\title{
Aseptic Meningitis Accompanied with Kikuchi-Fujimoto Disease
}

\author{
Hye Jin Hwang, MD', Eun Young Cho, MD', Yoon Young Yi, MD', Jin Man Kim, PhD², Joon Won Kang, PhD',3 \\ 'Department of Pediatrics, Chungnam National University Hospital, Daejeon, Korea \\ ${ }^{2}$ Department of Pathology, Chungnam National University Hospital, Chungnam National University College of Medicine, Daejeon, Korea \\ ${ }^{3}$ Department of Pediatrics, Chungnam National University College of Medicine, Daejeon, Korea
}

Received: July 16, 2019

Revised: August 20, 2019

Accepted: August 22, 2019

Corresponding author:

Joon Won Kang, PhD

Department of Pediatrics,

Chungnam National University

Hospital, Chungnam National

University College of Medicine,

282 Munhwa-ro, Jung-gu, Daejeon

35015, Korea

Tel: +82-42-280-7244

Fax: +82-42-255-3158

E-mail: childlove@cnu.ac.kr
Kikuchi-Fujimoto disease (KFD), also known as histiocytic necrotizing lymphadenitis, is a rare disease first reported in 1972 by Kikuchi [1] and Fujimoto et al. [2]. KFD is more common in young females under 30 years of age. It is a self-limited disease characterized by cervical lymphadenopathy. Headache accompanied by fever is a common symptom of this disease; however, the central nervous system (CNS) can also be involved. We describe a KFD patient with aseptic meningitis who had a headache as the first symptom of aseptic meningitis.

An 8-year-old boy presented with a 2-day history of fever and a nodule on the right side of the neck. At admission, he had a fever with a temperature of $38.5^{\circ} \mathrm{C}$. Physical examination showed multiple tender cervical lymph nodes with a swelling 2 to $3 \mathrm{~cm}$ in size on the right side of the neck. Neck movement was not limited. No abnormal neurological signs and symptoms were observed. The patient was discharged with a diagnosis of cervical lymphadenitis.

A week later, the patient was readmitted with fever and pain on the right side of the neck. There were no abnormal neurologic symptoms and neurological examination was normal. Laboratory tests on admission showed normal results. C-reactive protein level was $4.4 \mathrm{mg} / \mathrm{dL}$ (0 to 0.5 ). On the second day of readmission, a biopsy of the cervical lymph node was performed. The histopathology was consistent with KFD (Fig. 1). The patient was treated with supportive care including nonsteroidal anti-inflammatory drugs (NSAIDs) administration. General condition improved after 5 days and he was discharged.

Three days after the second discharge, he complained of fever and headache. The body temperature was $38.9^{\circ} \mathrm{C}$. The headache was in the forehead region, 6-point on the numerical pain rating scale, and alleviated with the administration of NSAIDs. No fever was observed after regular NSAIDs administration for every 8 hours. However, on the 6th day of admission, the headache aggravated severely, and the patient could not speak for a few seconds due to pain. Meningeal irritation signs were positive on neurological examination. The cerebrospinal fluid (CSF) opening pressure measured at lumbar puncture was $20 \mathrm{~cm}$ of water $\left(<18 \mathrm{~cm} \mathrm{H}_{2} \mathrm{O}\right)$. CSF was clear with a red blood cell count of $2 / \mu \mathrm{L}$, white blood cell count of $33 / \mu \mathrm{L}$ ( 0 to 5), glucose concentration of $67 \mathrm{mg} / \mathrm{dL}$ (simultaneously sampled blood sugar, $119 \mathrm{mg} / \mathrm{dL}$ ), plasma glucose ratio of 0.56 , and protein concentration of $47 \mathrm{mg} / \mathrm{dL}$ ( 15 to 45). CSF culture for bacteria was negative. Three days later, polymerase chain reaction for

Copyright(C) 2019 Korean Child Neurology Society

This is an Open Access article distributed under the terms of the Creative Commons Attribution Non-Commercial License (http://creativecommons.org/licenses/by-nc/4.0/) which permits unrestricted non-commercial use, distribution, and reproduction in any medium, provided the original work is properly cited. 


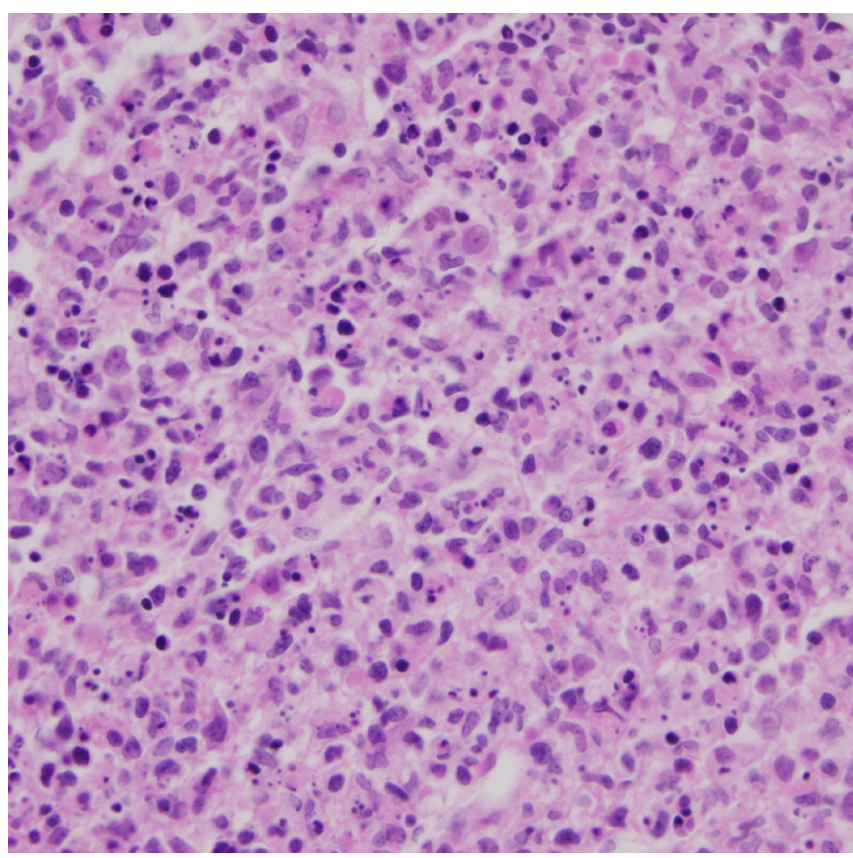

Fig. 1. Histopathology of the affected lymph node of the patient. Necrosis and abundant karyorrhectic debris with apoptosis are observed (hematoxylin and eosin stain, $\times 400$ ).

herpes simplex virus was reported as negative. On the 6th day of the third admission, magnetic resonance imaging of the brain with diffusion-weighted image showed no significant abnormalities. Since the CSF opening pressure was elevated, we started mannitol administration. After 3 days, the clinical symptoms improved and mannitol administration was stopped. Twelve days after the admission, the patient was discharged with no fever or headache. The lymph node size decreased to less than $1 \mathrm{~cm}$. Since discharge, the follow-up has been conducted for about a year in our outpatient clinic, and no identical symptoms have been observed.

KFD is a rare and self-limited disease. Due to its self-limiting nature, KFD is frequently misdiagnosed and comorbidities can be easily overlooked. About 5\% to $10 \%$ of the KFD patients showed central nervous CNS complications, which are resolved without any treatment $[3,4]$. Out of the $244 \mathrm{KFD}$ patients analyzed by Sato et al. [3], 11 patients (5\%) showed neurological involvement such as aseptic meningitis, mono-neuritis multiplex, hemiparesis, brachial neuritis, and photophobia.

Kucukardali et al. [4] reported aseptic meningitis in $9.8 \%$ of the
41 KFD patients studied. Noursadeghi et al. [5] reported a case of Kikuchi's disease with aseptic meningitis in a 37-year-old female and successfully treated it with corticosteroids alone. The authors suggested that a low dose of corticosteroid could shorten the duration of the illness. In our case, the patient's symptom relieved with mannitol only, but if the symptoms last even after mannitol administration, systemic steroid administration could be an option.

Although headache with fever is a common symptom in KFD, it can also be a symptom of meningitis. Given the fact that about $10 \%$ of the KFD cases show CNS involvement and that the disease is self-limited $[3,4]$, we could make an early decision for further evaluation and treatment of a KFD patient with a severe headache. In such cases, early consideration of the possibility of aseptic meningitis can be helpful in the assessment and in the timely treatment of the disease.

\section{Conflicts of interest}

No potential conflicts of interest relevant to this article was reported.

\section{ORCID}

Hye Jin Hwang, https:/ / orcid.org/0000-0002-7764-3085

Joon Won Kang, https://orcid.org/0000-0001-5756-3814

\section{References}

1. Kikuchi M. Lymphadenitis showing focal reticulum cell hyperplasia with nuclear debris and phagocytes: a clinicopathological study. Nihon Ketsueki Gakkai Zasshi 1972;35:379-80.

2. Fujimoto Y, Kojima Y, Yamaguchi K. Cervical subacute necrotizing lymphadenitis. Naika 1972;30:920-7.

3. Sato Y, Kuno H, Oizumi K. Histiocytic necrotizing lymphadenitis (Kikuchiss disease) with aseptic meningitis. J Neurol Sci 1999; 163:187-91.

4. Kucukardali Y, Solmazgul E, Kunter E, Oncul O, Yildirim S, Kaplan M. Kikuchi-Fujimoto disease: analysis of 244 cases. Clin Rheumatol 2007;26:50-4.

5. Noursadeghi M, Aqel N, Pasvol G. Kikuchi’s disease: a rare cause of meningitis? Clin Infect Dis 2005;41:e80-2. 